\title{
Evaluation of Form-Resistance of Fully-Formated Semi-Finished Furniture Sewing Products with Content of Polymer Composition
}

\author{
ZA Sabirova* and SSh Tashpulatov \\ Doctor of technical sciences, Tashkent Institute of Textile and Light Industry, Uzbekistan
}

Received: 䟧 June 19, 2018; Published: 眥 June 28, 2018

*Corresponding author: ZA Sabirova, Tashkent Institute of Textile and Light Industry Tashkent, Uzbekistan, Email: ziedasabirova@mail.rui

\begin{abstract}
The article presents the results of experimental studies on assessment, in particular, the substantiation of the use of polymer compositions and their application to the surface of a semi-finished product, which provides the desired form stability and reliability, meeting the modern requirements of industrial production, and the efficiency of their use in production conditions. In this case, the processing of all-seamed details of garments made from natural fur with the subsequent obtaining of a given shape by using the deformation properties of natural raw materials. As a result of the research, recommendations were given on the use of the proposed reinforcement options and the methods of the all-molded product package using a polymer composition, which makes it possible to improve the quality and shape stability of the garment details of their fur semi-finished products.
\end{abstract}

Keywords: Polymer Compositions; Deformation Properties; Shape Stability; Natural Fur; Whole Molded Products; Concentrated Load; Reliability of Results; Resource Saving

\section{Introduction}

It is known that fur products today are one of the main assortments of a modern man's wardrobe. At present, the deepest processing of natural raw materials to the finished product, the manufacture of garments from local raw materials, including natural fur, is given increased attention on the part of the state and is one of the key positions of the economic development program of the republic. In turn, high-quality manufacturing of finished products is a primary task of science and production. The index of the form-stability of parts of clothing is one of the main indicators of the quality of manufacturing of garments and is considered the most urgent task of science and production.

\section{Objects and Methods of Research}

Analysis of the data of scientific and technical information showed [1-3] that the most adequate indicator characterizing the form-stability of parts of clothing is the stability of a given shape to the impact of external, for example, concentrated force. To solve this problem, we use the technique proposed in [2], based on the use of the energy method [4]. Suppose that the volumetric form of the headdress made of natural fur in the section is closer to the spherical segment.

\section{Results and Discussion}

The figure shows the sections of the initial and deformed shape of the spherical segment under the influence of an external load, with the necessary geometric parameters before and after deformation.

Picture 1 Schematic diagram of the spherical segment: $P$ - applied concentrated external force; $W_{1}(r)$ - source segment; $W_{2}(r)$-deformed segment; $R$ - radius of the spherical segment; $H_{1}$ height of the initial spherical segment; $\mathrm{H}_{2}$ - height of the deformed spherical segment. 


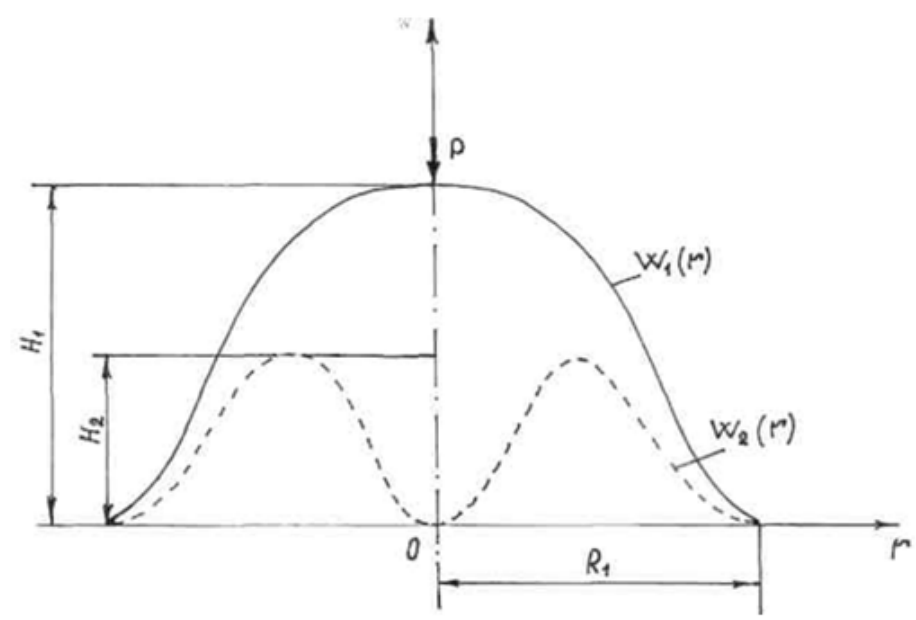

Picutre 1: Schematic diagram of the spherical segment: $P$ - applied concentrated external force; $W_{1}(r)$ - source segment; $R$ -deformed segment; $W_{2}(r)$ - radius of the spherical segment; $H_{1}$ - height of the initial spherical segment; $H_{2}$ - height of the deformed spherical segment.

All other things being equal, the value of the concentrated force will depend on the $P$ physico-mechanical properties of the sample packages and can serve as one of the indicators characterizing its form-stability.

The elastic curve of the initial section of the spherical $(W(r)$ segment can approximately be described by a cubic parabola [5]. Then

$$
\begin{aligned}
& W_{1}(r)=a_{1}+b_{1} r+c_{1} r^{2}+d_{1} r^{3} \\
& W_{1}^{I}(r)=b_{1}+2 c_{1} r+3 d_{1} r^{2}
\end{aligned}
$$

Where $a_{1}, b_{1}, c_{1}, d_{1}$ - are coefficients to be determined. They are based on the following conditions

$$
\left\{\begin{array}{l}
W_{1}(0)=H_{1}, \\
W_{1}\left(R_{1}\right)=0, \\
W_{1}^{I}(0)=0, \\
W_{1}^{I}\left(R_{1}\right)=0
\end{array}\right.
$$

Taking into account the boundary conditions (3), we have:

$$
\left\{\begin{array}{l}
a_{1}=H_{1}, \\
a_{1}+b_{1} R_{1}+c_{1} R_{1}^{2}+d_{1} R_{1}^{3}=0, \\
b_{1}=0, \\
b_{1}^{I}+2 c_{1} R_{1}+3 d_{1} R_{1}^{2}=0
\end{array}\right.
$$

and

$$
\left\{\begin{array}{l}
H_{1}+c_{1} R_{1}^{2}+d_{1} R_{1}^{3}=0 \\
2 c_{1} R_{1}+3 d_{1} R_{1}^{2}=0
\end{array}\right.
$$

Solving system (4) we have

$$
d_{1}=\frac{2 H_{1}}{R_{1}^{3}}, c_{1}=-3 \frac{H_{1}}{R_{1}^{2}}
$$

Consequently,

$$
W_{1}(r)=H_{1}-3 \frac{H_{1}}{R_{1}^{2}} r^{2}+\frac{2 H_{1}}{R_{1}^{3}} r^{3}
$$

Similarly, for a section of a deformed segment, one can write

$$
\begin{aligned}
& W_{2}(r)=a_{2}+b_{2} r+c_{2} r^{2}+d_{2} r^{3}, \\
& W_{2}^{I}(r)=b_{2}+2 c_{2} r+3 d_{2} r^{2}
\end{aligned}
$$

Where, $a_{2}, b_{2}, c_{2}, d_{2}$ - are coefficients to be determined, which are found from the following conditions

$$
\left\{\begin{array}{c}
W_{2}(0)=0, \\
W_{2}\left(R_{1}\right)=0, \\
W_{2}^{I}(0)=0, \\
W_{2}\left(\frac{R_{1}}{2}\right)=H_{2}
\end{array}\right.
$$

Substituting expressions (6) and (7) into conditions (8), we obtain

$$
\left\{\begin{array}{c}
a_{2}=0, \\
b_{2}=0, \\
c_{2} R_{1}^{2}+d_{2} R_{1}^{3}=0, \\
c_{2}\left(\frac{R_{1}}{2}\right)^{2}+d_{2}\left(\frac{R_{1}}{2}\right)^{3}=H_{2}
\end{array}\right.
$$

Solving the system of equations (9), we find the values $c_{2}, d_{2}$ :

$c_{2}=\frac{8 H_{2}}{R_{1}^{2}}, d_{2}=-\frac{8 H_{2}}{R_{1}^{3}}$

Then,

$W_{2}(r)=\frac{8 H_{2}}{R_{1}^{2}} r^{2}-\frac{8 H_{2}}{R_{1}^{3}} r^{3}(10)$

As a result, the deformation of a spherical segment can be described by equation

$$
\begin{aligned}
& W(r)=W_{1}(r)-W_{2}(r)=H_{1}-3 \frac{H_{1}}{R_{1}^{2}} r^{2}+\frac{2 H_{1}}{R_{1}^{3}} r^{3}-\frac{8 H_{2}}{R_{1}^{2}} r^{2}+\frac{8 H_{2}}{R_{1}^{3}} r^{3}= \\
& =H_{1}-\frac{1}{R_{1}^{2}}\left(3 H_{1}+8 H_{2}\right) r^{2}+\frac{1}{R_{1}^{3}}\left(2 H_{1}+8 H_{2}\right) r^{3}
\end{aligned}
$$

We denote by

$$
\begin{aligned}
& \frac{1}{R_{1}^{2}}\left(3 H_{1}+8 H_{2}\right)=k_{2}, \\
& \frac{1}{R_{1}^{3}}\left(2 H_{1}+8 H_{2}\right)=k_{3}
\end{aligned}
$$


then

$$
W(r)=H_{1}-k_{2} r^{2}+k_{3} r^{3}
$$

We use the energy method to determine $P_{l p}$. Assuming that the edges of the segment are pinched, the expression for the potential energy of the deformed spherical segment [5] is expressed in the form

$$
U=\int_{0}^{R_{1}} \int_{0}^{2 \pi}\left[\frac{D}{2}\left(\frac{\partial^{2} w}{\partial r^{2}}+\frac{1}{r} \frac{\partial w}{\partial r}\right)^{2}\right] r d r d \theta
$$

Then,

$W^{I}(r)=-2 k_{2} r+3 k_{3} r^{2}$,

$W^{I I}(r)=-2 k_{2}+6 k_{3} r$,

$\left[W^{I I}(r)+\frac{1}{r} W^{I}(r)\right]^{2}=\left(-2 k_{2}+6 k_{3} r-2 k_{2}+3 k_{3} r\right)^{2}=\left(-4 k_{2}+9 k_{3} r\right)^{2}=$

$=16 k_{2}-72 k_{2} k_{3} r+81 k_{3} r^{2}$,

$U=\int_{0}^{R_{1}} \int_{0}^{2 \pi}\left[\frac{D}{2}\left(16 k_{2}-72 k_{2} k_{3} r+81 k_{3} r^{2}\right)\right] r d r d \theta$

Integrating expression (14), we have

$U=\frac{\pi D}{R_{1}^{2}}\left(8 k_{2}^{2} R_{1}^{2}-24 k_{2} k_{3} R_{1}^{3}+\frac{81}{4} k_{3}^{2} R_{1}^{4}\right)$

Substituting expressions (11) and (12) into expression (15), we obtain the value of the potential energy

$$
\begin{aligned}
& U=\frac{\pi D}{R_{1}^{2}}\left[8\left(3 H_{1}+8 H_{2}\right)^{2}-24\left(3 H_{1}+8 H_{2}\right)\left(2 H_{1}+8 H_{2}\right)+\frac{81}{4}\left(2 H_{1}+8 H_{2}\right)^{2}\right]= \\
& =\frac{\pi D}{R_{1}^{2}}\left(9 H_{1}^{2}-72 H_{1} H_{2}+272 H_{2}^{2}\right),
\end{aligned}
$$

Here $D$ - is the stiffness of the specimen in bending

$$
D=\frac{E \delta^{3}}{12\left(1-v^{2}\right)}
$$

Where $v$ - is the coefficient of transverse contraction of the sample material? Finally, after the transformation, we have

$$
U=\frac{\pi E \delta^{3}}{12 R_{1}^{2}\left(1-v^{2}\right)}\left(9 H_{1}^{2}-72 H_{1} H_{2}+272 H_{2}^{2}\right)
$$

$P$

We determine the magnitude of the work of the force of gravity

$$
A=P H_{1}+\int_{0}^{2 \pi} \int_{0}^{R_{1}} W(r) q_{1} r d r d \theta,(17)
$$

Where $q$ - is the unit area weight?

Substituting the value into expression (17), we obtain

$$
A=P H_{1}+\int_{0}^{2 \pi} \int_{0}^{R_{1}}\left(H_{1}-k_{2} r^{2}+k_{3} r^{3}\right) q_{1} r d r d \theta
$$

Or taking into account (11), (12)

$$
\begin{aligned}
& A=P H_{1}+2 \pi q_{1}\left[H_{1} \frac{R_{1}^{2}}{2}-\frac{R_{1}^{2}}{4}\left(3 H_{1}+8 H_{2}\right)+\frac{R_{1}}{5}\left(2 H_{1}+8 H_{2}\right)\right]= \\
& =P H_{1}+2 \pi q_{1}\left(\frac{3}{20} R_{1}^{2} H_{1}-\frac{2}{5} R_{1}^{2} H_{2}\right)
\end{aligned}
$$

The value of $P$ the force is found from equality $U=A$

$$
P=\frac{\frac{\pi E \delta^{3}}{12 R_{1}^{2}\left(1-v^{2}\right)}\left(9 H_{1}^{2}-72 H_{1} H_{2}+272 H_{2}^{2}\right)-2 \pi q_{1}\left(\frac{3}{20} R_{1}^{2} H_{1}-\frac{2}{5} R_{1}^{2} H_{2}\right)}{H_{1}}
$$

The value of the critical force is determined in the range $0,637 \div 0,841 \mathrm{~N}$ with the para meters and the irvariations $\delta$
$=0,97 \cdot 10^{-3} \mathrm{~m} ; \mathrm{R}_{1}=0,15 \mathrm{~m} ; v=0,52 \div 0,55 ; \mathrm{H}_{1}=0,18 \mathrm{~m} ; \mathrm{H}_{2}=0,09 \div$ $0,11 \mathrm{~m} ; \mathrm{q}_{1}=4,3 \div 4,7 \mathrm{~N} / \mathrm{m}^{2}$. Experimental studies have determined the values of the critical force for deformation of bulk samples from natural fur - astrakhan, taking into account the application of the polymer composition in the package $[6,7]$. Convergence of the calculated and experimental data is completely satisfactory with a maximum relative error of no more than $10 \%$ [8].

\section{Conclusion}

The results of the carried out experiment confirm the dependence of the critical force, form stability and rigidity of the part package from application relative to the nominal axis of the semi-finished parts. Thus, the maximum value of the critical force $(0.841 \mathrm{H})$ is determined by applying the reinforcing polymer composition relative to the nominal fur axis at an angle of $\alpha=45^{\circ}$. At an angle of $\alpha=30^{\circ}$ and $60^{\circ}$ the critical force is $82,6 \%$ and $89,7 \%$ of the maximum value, respectively. The minimum value of the force $(75,8 \%$ of the maximum value) is determined in the packages with the application of the polymer composition at an angle of $\alpha$ $=0^{\circ}$ and $90^{\circ}$ relative to the nominal axis of the part. On the basis of the studies carried out, it is established principle possibility assessing of the shape stability and rigidity of bulk samples of a part by means of an analytical dependence that takes into account the physico-mechanical properties of materials and packages, as well as their design parameters taken into account in the formation of their package.

\section{References}

1. Melikov EH (1986) Working out and research of methods of forming of details of clothes: the author's abstract. Dis Doct. Sciences p. 42.

2. Tashpulatov SSh (2008) Development of highly effective resource-saving technology for the manufacture of garments: Abstract of thesis. Dis Doct tech Sciences p. 42.

3. Cherunova IV (2008) Theoretical bases of complex designing of special clothes: the author's abstract. Dis Doct tech Sciences Mines: YURGUES p. 42.

4. Timoshenko SP (1965) Resistance of materials. Nauka, Moscow, Russia 1: 364 .

5. Timoshenko SP, Voinovsky Criter S (1963) Plates and shells. State Publishing House of Physical and Mathematical Literature, Moscow, Russia pp. 635.

6. Tashpulatov SSh, Cherunova IV, Nutfullaeva LN (2015) The study of the form-stability of voluminous details of garments. Journal Student Scientific Forum Penza, Russia pp. 129-131.

7. Tashpulatov SSh, Nutfullaeva LN, Cherunova IV, Sten kina MP (2015) Hygienic assessment of the conditions of reinforcement of textile material of collagen-containing composition. Journal International Journal of Experimental Education. Moscow, Russia p. 415.

8. Sobirova ZA (2017) Geometric characteristics and change bulk form part of the product impregnated with the polymer composition based on collagen. International Journal of the European Science Review 1-2: 236-237. 

(C) (P) This work is licensed under Creative

To Submit Your Article Click Here: Submit Article

DOI: 10.32474/LTTFD.2018.02.000140

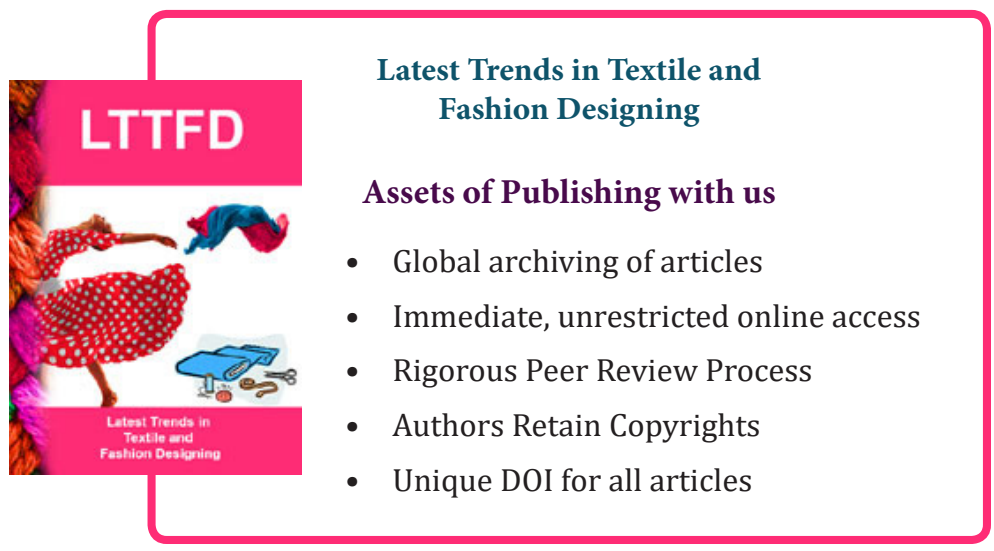

